${ }^{99 m}$ Tc-maraciclatide (Serac Healthcare) is a radiolabelled tracer which binds with high affinity to integrin $\alpha_{v} \beta_{3}$, a cell-adhesion molecule up-regulated on neoangiogenic blood vessels. It therefore has the potential to image synovial inflammation at the whole-body level. We previously showed in a pilot study that uptake was seen in the inflamed joints of five RA patients and that this correlated with PDUS. This study explores correlation with PDUS in a larger groups of patients with varied disease activity.

Objectives: To determine the correlation between ultrasound and ${ }^{99 \mathrm{~m}} \mathrm{Tc}-\mathrm{marac}-$ iclatide imaging in patients with rheumatoid arthritis.

Methods: 50 patients with RA fulfilling ACR 2010 classification criteria were recruited. Patients underwent an ultrasound scan of 40 joints with grey scale (GS) and PD quantification. Each joint was scored on a scale of 0-3 for GS and PD with a total score calculated for each patient. Within 3 hours of the ultrasound patients were injected with $740 \mathrm{MBq}$ of ${ }^{99 \mathrm{~m}} \mathrm{Tc}$-maraciclatide. Using a gamma camera, whole body planar views and dedicated hand and foot views were taken 2 hours after injection (Figure 1). Acquisition time was 20 minutes for whole body and 20 minutes for hand and foot views.

${ }^{99 \mathrm{~m}}$ Tc-maraciclatide images were scored as positive or negative uptake for each joint (binary score). A quantitative score was also calculated for each joint where there was uptake with this corrected for background uptake. Total binary and quantitative scores per patient were calculated.

Ultrasound and ${ }^{99 \mathrm{~m}} \mathrm{Tc}$-maraciclatide scores were tested for correlation with Pearson's correlation coefficient ( $r$ ). Interrater agreement for 2 scorers was calculated using kappa $(\mathrm{k})$ and concordance correlation coefficient $(P C)$.

Results: Strong correlation was seen when total PDUS was compared to binary scores $\left(r=0.92, r^{2}=0.85\right)$ (Figure 2) and quantitative scores $\left(r=0.85, r^{2}=0.72\right) . k$ was 0.82 and 0.79 for binary and ultrasound scores respectively. $P C$ was 0.82 for quantitative scores. p was $<0.0005$ for all results. ${ }^{99 \mathrm{~m}} \mathrm{Tc}$-maraciclatide uptake was also seen in inflamed tendons/tendon sheaths. The imaging procedure was well-tolerated. There were no tracer-related adverse events.

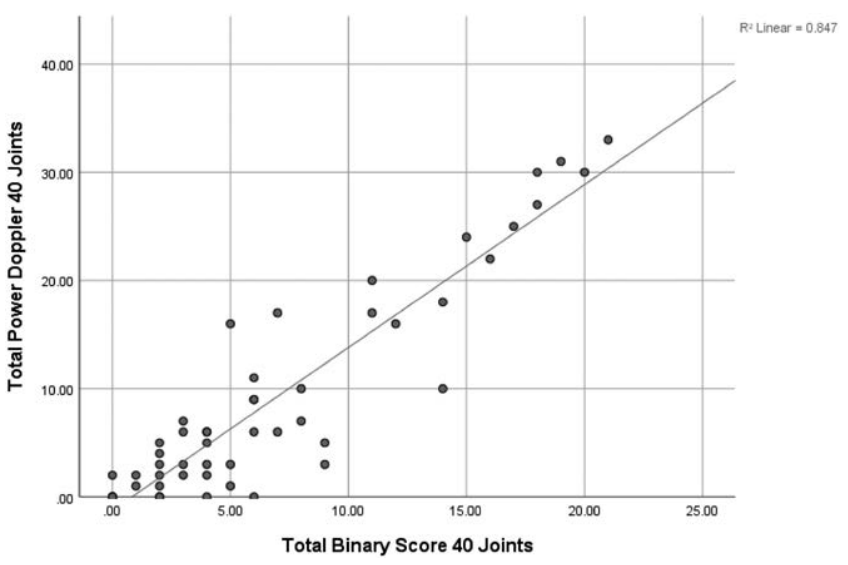

Figure 1. ${ }^{99 \mathrm{~m}} \mathrm{Tc}$-maraciclatide imaging with dedicated hand and foot views

Conclusion: ${ }^{99 \mathrm{~m}} \mathrm{Tc}$-maraciclatide uptake was highly correlated with PDUS highlighting its potential as an alternative imaging modality. ${ }^{99 \mathrm{~m}} \mathrm{Tc}$-based planar imaging has the unique capacity to image the whole body and hence the total synovial inflammatory load in a quick acquisition. The imaging equipment to perform these scans is already widely available in radiology departments. Interpretation of scans is also much simpler compared to US/MRI. It could therefore have a role in key decision-making points in pathways for diagnosis, treatment failure, and remission prior to dose tapering.

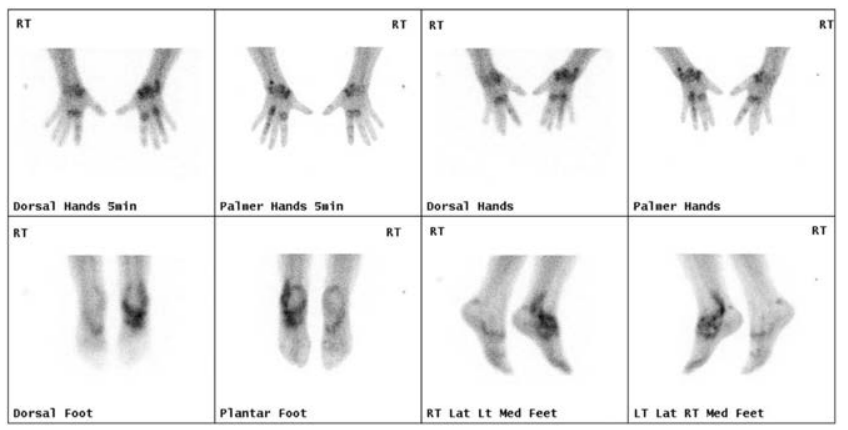

Figure 2. Correlation between total power doppler and ${ }^{99 \mathrm{~m}} \mathrm{Tc}$-maraciclatide binary scores
Disclosure of Interests: None declared

DOI: 10.1136/annrheumdis-2020-eular.5482

\section{OP0179 \\ USEFUL STUDY I: A MULTICENTRE LONGITUDINAL STUDY TO TEST WHETHER ULTRASOUND CAN IDENTIFY PATIENTS WITH MUSCULOSKELETAL SYMPTOMS OF LUPUS WITH BETTER RESPONSE TO THERAPY}

K. Mahmoud ${ }^{1}$, A. Zayat ${ }^{2}$, M. Y. MD Yusof ${ }^{1}$, K. Dutton ${ }^{1}$, L. S. Teh ${ }^{3}$, C. S. Yee ${ }^{4}$, D. D'cruz ${ }^{5}$, N. Ng ${ }^{5}$, D. Isenberg ${ }^{6}$, C. Ciurtin ${ }^{6}$, P. G. Conaghan ${ }^{1}$, P. Emery ${ }^{1}$, C. Edwards ${ }^{7}$, E. Hensor ${ }^{1}$, E. Vital ${ }^{1} .{ }^{1}$ University of Leeds and NIHR Leeds Biomedical Research Centre, Leeds, United Kingdom; ${ }^{2}$ Bradford Teaching Hospitals NHS Foundation Trust, Bradford, United Kingdom; ${ }^{3}$ Royal Blackburn Teaching Hospital and University of Central Lancashire, Blackburn, United Kingdom; ${ }^{4}$ Doncaster and Bassetlaw NHS Trust, Doncaster, United Kingdom; ${ }^{5}$ Guys and St Thomas Hospital, London, United Kingdom; ${ }^{6}$ University College London, London, United Kingdom; ${ }^{7}$ University Hospital Southampton NHS Foundation Trust, Southampton, United Kingdom

Background: In SLE, musculoskeletal manifestations impact on quality of life and trial outcomes. We previously showed that assessments based on joint swelling lack sensitivity, specificity and responsiveness compared to ultrasound (US).

Objectives: To determine clinical features predicting US synovitis and whether patients with US synovitis respond better to therapy

Methods: SLE patients were recruited if the referring physician deemed they had inflammatory pain warranting treatment. Swollen joints were not required. At baseline, physicians recorded features of inflammation, concurrent fibromyalgia and osteoarthritis. Stable doses of prednisolone ( $\leq 5 \mathrm{mg} /$ day), antimalarials or immunosuppressants were allowed. Participants received depomedrone 120mg IM then were assessed at 0,2 and 6 weeks for $66 / 68$ swollen and tender joint counts, BILAG-2004, SLEDAI-2K, physician global and MSK-VAS, inflammatory markers, patient pain and disease activity-VAS, HAQ-DI, LupusQoL, US of hands and wrists (blinded to patient and clinical assessor). An internal pilot determined the primary endpoint:(Early Morning stiffness-VAS (EMS-VAS) at 2 weeks (adjusted for baseline) between patients with US-synovitis (GS $\geq 2$ or $P D \geq 1$ in $\geq 1$ joint) vs. normal US at baseline. $20 \%$ difference was considered clinically meaningful. Sensitivity analyses adjusted for prednisolone and immunosuppressants. Results: 122/133 patients completed all visits. There was significant disagreement between clinical examination and US. $78 / 133$ had US synovitis; $68 \%$ of these had $\geq 1$ swollen joint. Of $66 / 133$ patients with $\geq 1$ swollen joint, $20 \%$ had normal US. US-synovitis was more likely with joint swelling, a symmetrical smal joint distribution and active serology. Physician-determined EMS, other lupus features or prior response to therapy were not associated. Fibromyalgia or osteoarthritis did not reduce the probability of US synovitis.

In the full analysis set $(n=133)$ there was no difference in EMS VAS at 2 weeks according to US synovial status at baseline (difference $-8 \mathrm{~mm}, 95 \% \mathrm{Cl}-19,4 \mathrm{~mm}$, $p=0.178)$. 32 patients had fibromyalgia. After excluding them, we found a statistically and clinically significantly better clinical response to depomedrone in patients with US-synovitis at baseline (baseline-adjusted EMS VAS at 2 weeks $-12 \mathrm{~mm}, 95 \% \mathrm{Cl}-24,0 \mathrm{~mm}, \mathrm{p}=0.049)$. This difference was greater in the treatment-adjusted sensitivity analysis $(-12.8(95 \% \mathrm{Cl}-22,-3 \mathrm{~mm}), \mathrm{p}=0.007)$ and the per-protocol-adjusted sensitivity analysis $(-14.8 \mathrm{~mm}(95 \% \mathrm{Cl}-20.8,-8.8 \mathrm{~mm})$ $\mathrm{p}<0.001)$. Patient with US synovitis had higher rates of improvement in the MSK BILAG-2004 (56\% vs. $26 \%, p=0.09$ ) and SLEDAI-2K (37\% vs. 15\%, $p=0.03$ ).

Conclusion: In lupus arthritis, distribution and serology, but not other features, help identify US-synovitis. US-synovitis was independent of features of fibromyalgia, but fibromyalgia confounded assessment of clinical response. US should be used to select SLE arthritis patients for therapy and clinical trials, especially when there are inflammatory symptoms without swollen joints.

Acknowledgments: The Project was funded by Lupus-UK

Disclosure of Interests: Khaled Mahmoud: None declared, Ahmed Zayat: None declared, Md Yuzaiful Md Yusof: None declared, Katherine Dutton: None declared, Lee-Suan Teh: None declared, Chee-Seng Yee: None declared, David d'cruz Grant/research support from: GlaxoSmithKline, Nora Ng: None declared, David Isenberg Consultant of: Study Investigator and Consultant to Genentech, Coziana Ciurtin Grant/research support from: Pfizer, Consultant of: Roche, Modern Biosciences, Philip G Conaghan Consultant of: AbbVie, BMS, Eli Lilly, EMD Serono, Flexion Therapeutics, Galapagos, GSK, Novartis, Pfizer, Speakers bureau: AbbVie, Eli Lilly, Novartis, Pfizer, Paul Emery Grant/research support from: AbbVie, Bristol-Myers Squibb, Merck Sharp \& Dohme, Pfizer, Roche (all paid to employer), Consultant of: AbbVie (consultant, clinical trials, advisor), Bristol-Myers Squibb (consultant, clinical trials, advisor), Lilly (clinical trials, advisor), Merck Sharp \& Dohme (consultant, clinical trials, advisor), Novartis (consultant, clinical trials, advisor), Pfizer (consultant, clinical trials, advisor), Roche (consultant, clinical trials, advisor), Samsung (clinical trials, advisor), Sandoz (clinical trials, advisor), 
UCB (consultant, clinical trials, advisor), Christopher Edwards Grant/research support from: Abbvie, Biogen, Roche, Consultant of: Abbvie, Samsung, Speakers bureau: Abbvie, BMS, Biogen, Celgene, Fresenius, Gilead, Janssen, Lilly, Mundipharma, Pfizer, MSD, Novartis, Roche, Samsung, Sanofi, UCB, Elizabeth Hensor: None declared, Edward Vital Grant/research support from: AstraZeneca, Roche/ Genentech, and Sandoz, Consultant of: AstraZeneca, GSK, Roche/Genentech, and Sandoz, Speakers bureau: Becton Dickinson and GSK DOI: 10.1136/annrheumdis-2020-eular.2160

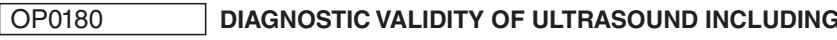 EXTRA-CRANIAL ARTERIES IN GIANT CELL ARTERITIS}

I. Monjo ${ }^{1}$, E. Fernández ${ }^{1}$, D. Peiteado ${ }^{1}$, A. Balsa ${ }^{1}$, E. De Miguel ${ }^{1} .{ }^{1}$ University Hospital La Paz, Rheumatology, Madrid, Spain

Background: Giant cell arteritis (GCA) is a chronic vasculitis of the medium and large arteries. The involvement of large vessel (LV) either isolated or associated with cranial artery is frequent, so it is necessary to use imaging techniques for diagnosis, because the biopsy in these cases is not useful. European League Against Rheumatism (EULAR) recommends an early imaging test in patients with suspected GCA, and ultrasound of temporal \pm axillary arteries is recommended as the first imaging modality in patients with suspected predominantly cranial GCA (1).

Objectives: To assess the validity of Colour Doppler ultrasound (CDUS) of temporal superficial arteries (TA) and LV (axillary, subclavian and carotid) in the diagnosis of GCA, using as gold standard the patient's definitive clinical diagnosis. Analyse if routine ultrasound examination of LV improves the diagnostic accuracy.

Methods: This was an observational, descriptive and analytical study of 198 consecutive patients with GCA suspicion. A baseline CDUS of the TA and LV was performed. Ultrasound diagnosis was made according to the OMERACT (Outcome Measures in Rheumatology) definitions of halo sign and was established as a limit of average intimal thickness $\geq 0.34 \mathrm{~mm}$ for superficial temporal arteries and $\geq 1 \mathrm{~mm}$ for axillary, subclavian and carotid arteries. Statistical analysis was performed using SPSS version 25

Results: Eighty-seven patients (43.9\%) were CDUS compatible with GCA, and 111 patients $(56.1 \%)$ had a negative CDUS. Among the patients with positive CDUS three different patterns were detected: 45 patients $(51.7 \%)$ had an exclusive cranial involvement, $31(35.6 \%)$ had a mixed pattern with involvement of both TA and LV and 11 (12.6\%) had an exclusive LV involvement. The validity (sensitivity and specificity) and security (positive predictive value and negative predictive value) of diagnostic are shown in table.

When we analyse patients with LV involvement, $87.8 \%$ have axillary artery involvement, $77.4 \%$ subclavian involvement and $34.4 \%$ carotids involvement. If we only explored the axillary arteries, $12.2 \%$ of patients with LV involvement would not be diagnosed. However, if we explored axillary and subclavian arteries, $100 \%$ of patients with LV involvement would be diagnosed.

Conclusion: Half of the patients with GCA have LV involvement and up to $12.8 \%$ exclusively LV affectation in our series. Adding CDUS exploration of LV arteries to TA increases both sensitivity and diagnostic specificity. The minimum ultrasound examination of LV should include both axillary and subclavian arteries. References:

[1] Dejaco C, Ramiro S, Duftner C, et al. EULAR recommendations for the use of imaging in large vessel vasculitis in clinical practice. Ann Rheum Dis. 2018;77(5):636-3

\begin{tabular}{lcccc}
\hline & Sensitivity & Specificity & $\begin{array}{c}\text { Positive } \\
\text { predictive value }\end{array}$ & $\begin{array}{c}\text { Negative } \\
\text { predictive value }\end{array}$ \\
\hline CDUS TA and LV & $97,7 \%$ & $97,3 \%$ & $96,6 \%$ & $98,2 \%$ \\
CDUS TA & $83,9 \%$ & $97,3 \%$ & $96,1 \%$ & $88,5 \%$ \\
\hline
\end{tabular}

Disclosure of Interests: Irene Monjo: None declared, Elisa Fernández: None declared, Diana Peiteado: None declared, Alejandro Balsa Grant/research support from: BMS, Roche, Consultant of: AbbVie, Gilead, Lilly, Pfizer, UCB, Sanofi,
Sandoz, Speakers bureau: AbbVie, Lilly, Sanofi, Novartis, Pfizer, UCB, Roche, Nordic, Sandoz, Eugenio de Miguel Grant/research support from: Yes (Abbvie Novartis, Pfizer), Consultant of: Yes (Abbvie, Novartis, Pfizer), Paid instructo for: yes (AbbVie, Novartis, Pfizer, MSD, BMS, UCB, Roche, Grunental, Janssen, Sanofi), Speakers bureau: yes (AbbVie, Novartis, Pfizer, MSD, BMS, UCB Roche, Grunental, Janssen, Sanofi) DOI: 10.1136/annrheumdis-2020-eular.5036

\section{OP0181 FUNCTIONAL CUT-OFFS TO DISTINGUISH PULMONARY VASCULAR AND PARENCHYMAL INVOLVEMENT IN SYSTEMIC SCLEROSIS (SSC): A QUANTITATIVE ANALYSIS OF IMAGING FEATURES AT CHEST COMPUTED TOMOGRAPHY (CT)}

C. Bruni ${ }^{1}$, M. Occhipinti ${ }^{2,3}$, G. Camiciottoli ${ }^{3}$, M. Bartolucci ${ }^{4}$, G. Lepri ${ }^{1}$,

A. Fabbrizzi ${ }^{3}$, A. Tottoli ${ }^{1}$, A. Bassetto ${ }^{3}$, G. Ciardi ${ }^{3}$, D. Giuggioli ${ }^{5}$, G. Cuomo ${ }^{6}$, F. Masini ${ }^{6}$, F. Lavorini ${ }^{3}$, L. Calistri ${ }^{4}$, M. Matucci-Cerinic ${ }^{1} .{ }^{1}$ University of Florence, Rheumatology, Florence, Italy; ${ }^{2}$ University of Florence, Radiology, Firenze, Italy; ${ }^{3}$ University of Florence, Pulmonology, Firenze, Italy; ${ }^{4}$ Careggi University Hospital, Radiology, Firenze, Italy; ${ }^{5}$ Policlinico of Modena University Hospital of Modena, Rheumatology, Modena, Italy; ${ }^{6}$ University of Campania Studies "Luigi Vanvitelli", Napoli, Italy

Background: Interstitial lung disease (ILD) and pulmonary arterial hypertension represent the most frequent causes of morbidity and mortality in SSc, with chest CT representing the gold standard in ILD assessment, while FVC and DLco allow functional assessment.

Objectives: As qualitative analysis of given chest CT scans is hampered by low reproducibility, we aimed to perform a quantitative analysis (QA) of CT scans able to investigate the parenchymal and vascular features in SSc-ILD and thus testing the relationship with clinical-functional data.

Methods: We prospectively enrolled 80 patients who underwent PFTs and spirometry-gated chest CT scan at TLC on the same day. Clinical, lung functional and diffusion data, as well as disability indexes were collected. CT images were analyzed by a computational platform for texture analysis of ILD patterns (CALIPER) through Imbio LTA. It quantified the extent of normal lung $(\% \mathrm{~N})$, groundglass opacities (\%GG), reticulation (\%RET), honeycombing (\%HC), hyperlucent $(\% \mathrm{HL})$, absolute (PVV, $\mathrm{cm}^{3}$ ) and normalized (PVV/LV, \%) pulmonary vascular volumes. Cut-offs of normality for \%FVC and \%DLco of $80 \%$ and $70 \%$ were tested to differentiate parenchymal and vascular features.

Results: 73 patients/CT scans were eligible for both software analyses. CALIPER showed GG\% as the most frequent radiological pattern (mean $5.5 \pm 10.4 \%$ ). $\%$ FVC and \% TLC negatively correlated with all ILD patterns, while \%DLco with RET\% only; PVV and PVV/LV negatively correlated with \%FVC and \%TLC, while $\%$ DLco with PVV/LV only. Positive correlations were found between all ILD patterns and vascular volumes (Table 1)

\begin{tabular}{lccccccccc}
\hline & & LV $\left(\mathrm{cm}^{3}\right)$ & $\% \mathrm{~N}$ & $\% \mathrm{GG}$ & $\% \mathrm{RET}$ & $\% \mathrm{HC}$ & $\% \mathrm{HL}$ & $\mathrm{PVV}\left(\mathrm{cm}^{3}\right)$ & $\%$ PVV/LV \\
\hline $\mathrm{FVC} \%$ & $\mathrm{r}$ & .60 & -.19 & -.40 & -.34 & -.30 & .35 & -.26 & -.44 \\
& $\mathrm{p}$ & $<.001$ & - & $<.001$ & .004 & .01 & .003 & .04 & $<.001$ \\
$\mathrm{FEV}_{1} \%$ & $\mathrm{r}$ & .58 & -.02 & -.38 & -.25 & -.24 & .23 & -.35 & -.49 \\
& $\mathrm{p}$ & $<.001$ & - & .002 & .04 & .05 & - & .004 & $<.001$ \\
$\mathrm{FEV}_{1} / \mathrm{FVC}$ & $\mathrm{r}$ & -.16 & .33 & .22 & .16 & .21 & -.35 & -.15 & -.08 \\
& $\mathrm{p}$ & - & .02 & - & - & - & - & - & - \\
$\mathrm{TLC} \%$ & $\mathrm{r}$ & .71 & -.14 & -.42 & -.37 & -.48 & .40 & -.43 & -.64 \\
& $\mathrm{p}$ & $<.001$ & - & .001 & .01 & $<.001$ & .002 & $<.001$ & $<.02$ \\
DLCO\% & $\mathrm{r}$ & .38 & -.05 & -.21 & -.31 & -.22 & .30 & -.21 & -.33 \\
& $\mathrm{p}$ & .01 & - & - & .01 & - & - & - & .006 \\
FVC/DLCO & $\mathrm{r}$ & .03 & -.08 & -.06 & -.003 & -.09 & .08 & -.06 & -.08 \\
& $\mathrm{p}$ & - & - & - & - & - & - & - & - \\
\hline
\end{tabular}

Cut-offs equal to 80 for \%FVC and 70 for \%DLco distinguished both parenchymal and vascular features, while 80 for \%DLco characterized vascular features only. These results were confirmed also when patients were stratified according to absent/single/combined \%FVC and $\%$ DLCO impairments with $80 \%$ cut-offs (Table 2 ).

Conclusion: In SSc a cut-off at 80 for \%DLco may help identifying vascular changes as automatically assessed on chest CT scan, without any underlying

\begin{tabular}{|c|c|c|c|c|c|c|c|c|c|}
\hline & $\mathrm{FVC}<80 \%$ & $\mathrm{FVC} \geq 80 \%$ & $p$ & DLco $<80 \%$ & DLco $\geq 80 \%$ & $p$ & DLco $<70 \%$ & DLco $\geq 70 \%$ & $p$ \\
\hline$\% \mathrm{~N}$ & $82.7(9.6)$ & $86.2(14.7)$ & - & $86.6(12.7)$ & $80.8(15.8)$ & - & $84.1(13.9)$ & $86.4(13.5)$ & - \\
\hline$\% G G$ & $10.3(8.9)$ & $2.4(3.9)$ & $<.001$ & $5.0(6.7)$ & $3.9(6.9)$ & - & $6.2(7.5)$ & $2.4(4.8)$ & .002 \\
\hline \%RET & $2.9(2.9)$ & $0.8(1.3)$ & $<.001$ & $1.6(2.1)$ & $0.7(0.9)$ & - & $1.9(2.4)$ & $0.6(0.8)$ & .007 \\
\hline$\% \mathrm{HC}$ & $0.4(0.6)$ & $0.1(0.1)$ & $<.001$ & $0.2(0.3)$ & $0.1(0.1)$ & - & $0.2(0.4)$ & $0.05(0.2)$ & .010 \\
\hline$\% \mathrm{HL}$ & $3.6(6.8)$ & 8.9 (12.1) & - & $5.4(8.8)$ & $14.1(15.4)$ & .050 & $6.3(10.1)$ & $9.2(12.7)$ & - \\
\hline PVV & $125.6(39.1)$ & 90.9 (26.9) & $<.001$ & 101.9 (34.8) & $84.7(19.4)$ & .016 & $106.9(38.3)$ & $87.5(20.5)$ & .012 \\
\hline PVV/LV & $3.8(1.6)$ & $2.0(0.7)$ & $<.001$ & $2.51(1.3)$ & $1.7(0.6)$ & .002 & $2.76(1.4)$ & $1.83(0.6)$ & .001 \\
\hline
\end{tabular}

